\title{
BILATERAL OVARIAN TORSION: A CASE REPORT
}

Manohar R ${ }^{1}$, Gopalakrishna K. H², Kavyashree G³ ${ }^{3}$ Kala B ${ }^{4}$, Karthik B ${ }^{5}$

\section{HOW TO CITE THIS ARTICLE:}

Manohar R, Gopalakrishna K. H, Kavyashree G, Kala B, Karthik B. "Bilateral Ovarian Torsion: A Case Report". Journal of Evolution of Medical and Dental Sciences 2014; Vol. 3, Issue 47, September 25; Page: 11472-11476, DOI: $10.14260 /$ jemds/2014/3502

ABSTRACT: Bilateral ovarian torsion is a rare event with only a few cases reported in literature in women using ovarian stimulation drugs and in pre-menarchal age group with synchronous and asynchronous ovarian tumors. We hereby present a case of bilateral ovarian torsion in a 16 year old girl who presented as an acute pain abdomen and was diagnosed intra operatively.

KEYWORDS: Ovary, torsion, ovarian, ovariotomy, cyst.

INTRODUCTION: Acute ovarian torsion is an uncommon cause of pain abdomen in children which is frequently confused with other conditions and having a potential life-long consequence for fertility. ${ }^{1}$

Bilateral ovarian torsion is a rare event with only a few cases reported in literature in women using ovarian stimulation drugs and in pre-menarchal age group with synchronous and asynchronous ovarian tumors. ${ }^{2}$

CASE REPORT: Miss XX 16 year old presented with pain abdomen since 1 day to OPD. Pain was localized to the left lumbar region, intermittent, non-radiating and associated with two episodes of vomiting since morning. Vomitus was non-projectile, non-bile stained, not associated with hematemesis and non-foul smelling. She gave a history of appendicectomy 6 years back. She was unmarried. Her previous cycles were regular for every 28-30 days and duration of menstrual flow was for 3-4 days and not associated with passage of clots or dysmenorrhea.

On general physical examination she was afebrile, pulse rate was 90 per minute, good volume blood pressure was 100/60 mm of Hg. Her cardiovascular system and respiratory system were normal. On per abdomen examination tenderness was present in both iliac fossae.

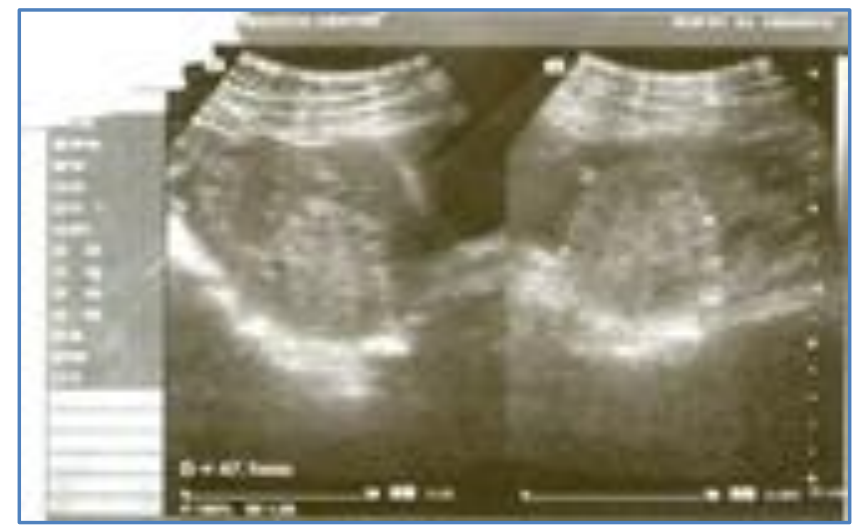

Left ovarian enlargement $7.7 * 5.1 \mathrm{cms}$ and echogenicity appeared normal. Doppler study showed no flow in the left ovary. Right ovary was normal in shape and echogenicity. Minimal free fluid noted in the POD 


\section{CASE REPORT}

An emergency ultrasound was done on admission which revealed:

Impression: enlarged left ovary? Inflammatory.

ON ADMISSION: Patient was kept nil orally for 24 hours. Inj taxim $1 \mathrm{~g} \mathrm{IV}$ bd after test dose was given (as ultrasound showed inflamed ovary). Patient's symptoms subsided within 24 hours. Repeat ultrasound was done at another centre, which revealed:

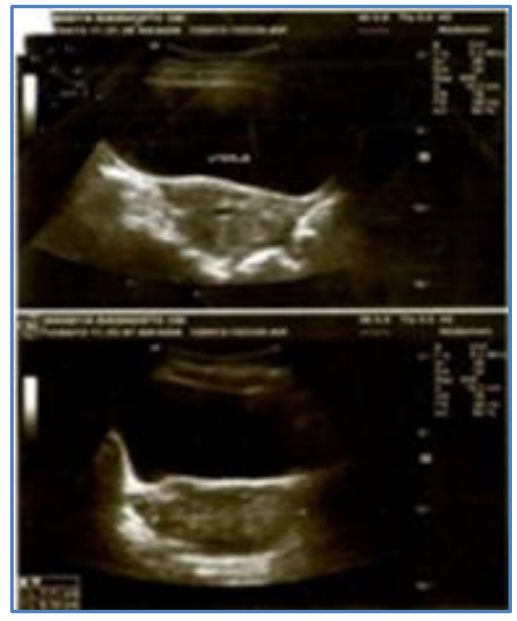

Right ovary enlarged measures $8 * 5 * 4.7 \mathrm{cms}$, solid in nature, Doppler study showed no obvious vascular flow. Culde sac: very minimal fluid collection seen. Impression: Right sided ovarian torsion

Under sub arachnoid block, emergency laparotomy was preceded with left salpingooophorectomy with right ovariotomy.

INTRA-OPERATIVE FINDINGS: Hemoperitoneum $100 \mathrm{ml}$. Left sided adnexal torsion $8 * 10 \mathrm{cms}$, edematous congested and gangrenous ovary and fallopian tube.

Right sided torsion ovary $5^{*} 4 \mathrm{cms}$ greenish colored (torsion one and half turns clockwise). Uterus was normal size.
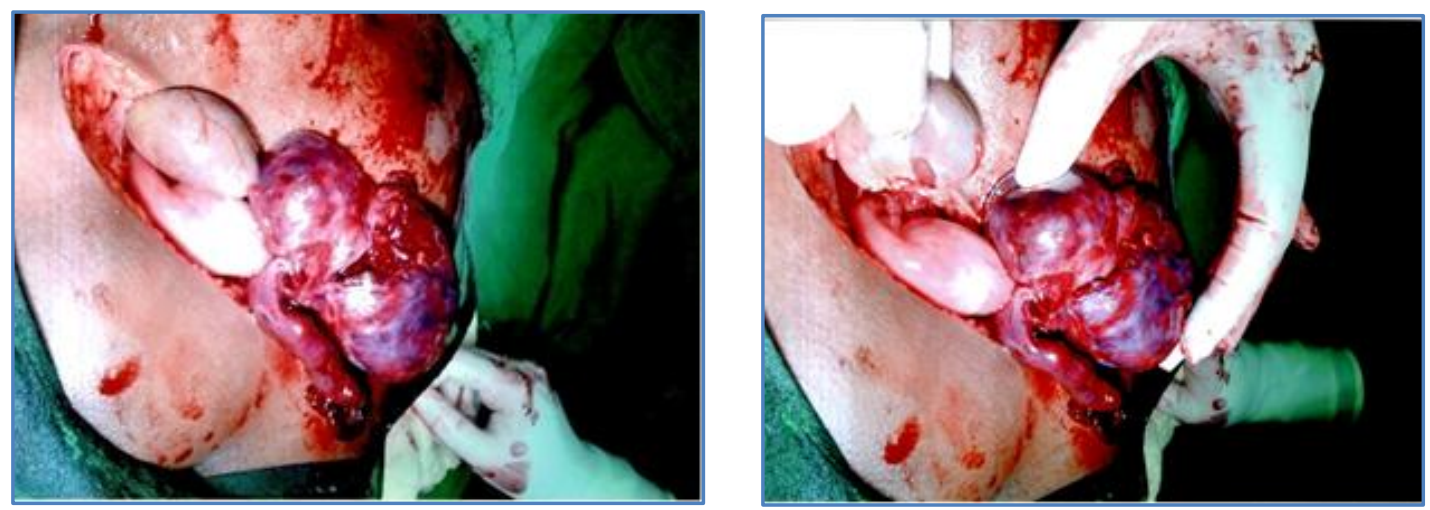


\section{HISTOPATHOLOGY REPORT:}

A. Left ovary: Gross: a specimen consist of dusky red oval mass of tissue (congested ovary) sent. With irregular sections and fallopian tube stretched across. Ovary measuring $9 * 6 * 3 \mathrm{~cm}$, fallopian tube measures $7 \mathrm{~cm}$ in length. Ovary cut section is dark brown. Fallopian tube is enlarged, cut sections show blood clots.

\section{Microscopy:}
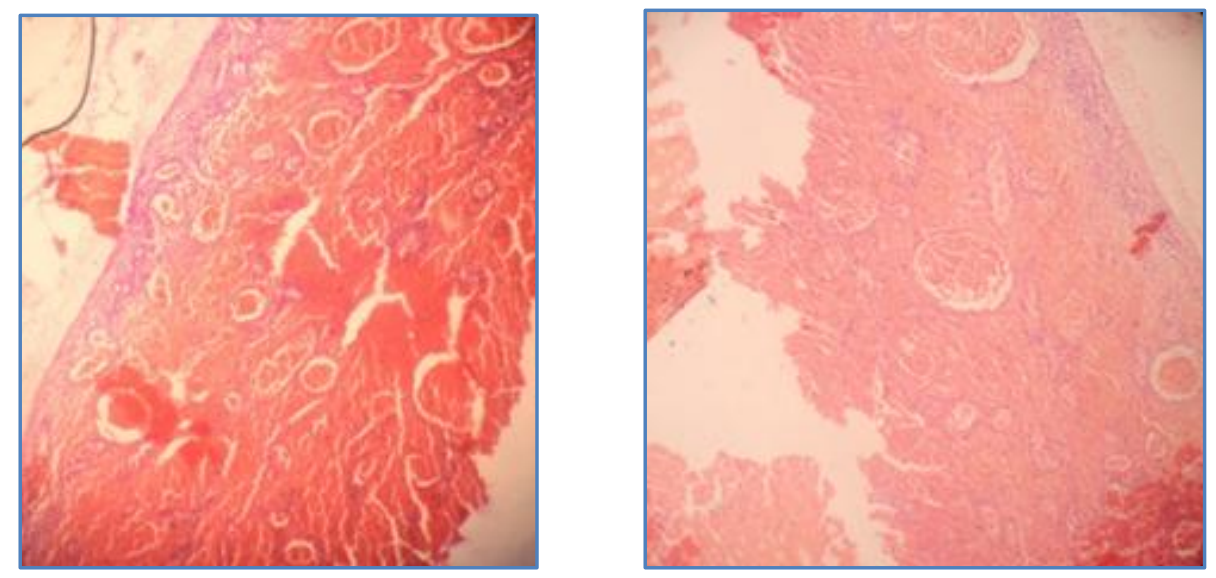

Ovarian cyst wall showing extensive hemorrhage distended and stretched out fallopian tube With necrosis -100x magnification showing features of hematosalphinx - 100x

\section{B. Right ovary:}

Gross: Specimen consists of ovary sent section measuring $4 * 3 * 2 \mathrm{~cm}$. cut sections shows reddish friable material.

Microscopy: Sections from the ovary shows haemorrhagic infraction.

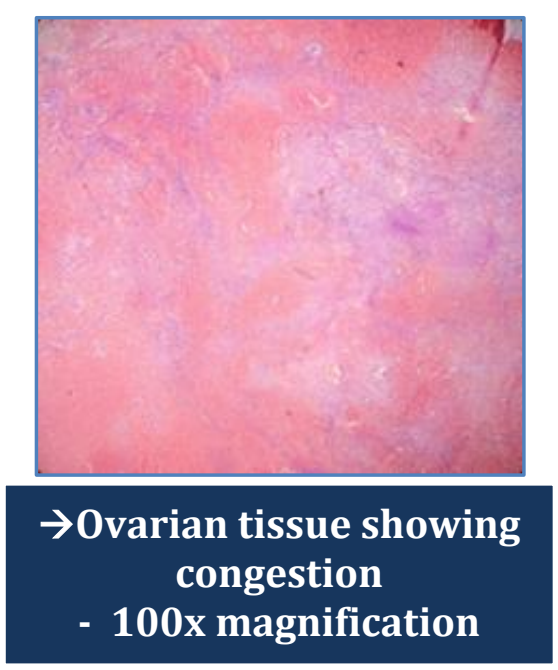

Impression: Hemorrhagic infarction b

/l ovaries 
DISCUSSION: Acute ovarian torsion accounts for up to $2.7 \%$ of all cases with acute abdominal pain in children and is the most common complication of ovarian tumors in children with an incidence ranging from $3 \%-16 \% .{ }^{1}$ Normal ovaries can be associated with up to $20-25 \%$ of cases with acute ovarian torsion. Normal ovaries in addition to ovaries with benign pathology are responsible for 61$97 \%$ of AOT. 1

Ovarian torsion occurs secondary to the abnormal twisting of the involved ovary on its ligamentous support. Torsion of the ovarian blood supply will result in venous congestion, hemorrhage, and eventually ischemia. Prolonged ischemia of the ovary or the adnexal structures can lead to necrosis, resulting in loss of ovarian function or infection or peritonitis.

The anatomy is such that the right side is more frequently affected than the left. In pediatric patients, ovarian torsion can be caused by a variety of anatomic mechanisms. Most studies have found approximately $50 \%$ of ovarian torsion in pediatric patients to involve adnexal cysts, teratomas, or other benign masses, including polycystic ovaries. ${ }^{3}$

MRI and CT can also reliably detect ovarian lesions and have been recommended as adjunctive diagnostic modalities. ${ }^{4}$ Laparoscopic surgery, with its benefits of less blood loss, less pain and faster recovery is the ideal route for adnexal surgery. Recent literature suggests a conservative line of management by de-torsion of the torsed ovaries, as vascularity can be regained even in the ischemic ovaries and thereby conservation of the ovaries in view of future fertility purpose. 5

In this particular case, diagnosis of unilateral ovarian torsion was made by sonography and by laparotomy bilateral ovarian torsion was made and was proceeded with bilateral ovariotomy as both ovaries were non-viable and after taking risk consent at the time of surgery. Currently the patient is on regular follow-up with hormone replacement therapy and her coagulation profile Liver function test and hematological tests are normal. However, we deeply regret for the surgery as recent literature favors ovarian detorsion and conservation of ovaries.

CONCLUSION: Bilateral ovarian torsion is a rare event and can present as acute pain abdomen in young patients. Imaging modalities like CT and MRI maybe beneficial in diagnosing bilateral ovarian torsion in pediatric patients pre-operatively. However in view of future fertility conservation of the ovary by de-torsion has to be attempted in all cases.

\section{REFERENCES:}

1. Dolunay Alver, Cengiz Gul, Aysenur Cerrah Celayir, Davut Sahin. A case study of ovarian torsion with a serous cyst and co-existing serous cystadenoma in the contralateral ovary. Journal of Pediatric Surgical Specialities. www.jpss.eu Volume 3 NO 3 NO 1.

2. Kitporntheranunt M, Wong J, Siow A. Entangled bilateral adnexal torsion in a premenarchal girl: a laparoscopic approach. Singapore Med J 2011; 52 (6): e124.

3. Dumont T, Caccia N, Allen L. Pediatric synchronous bilateral ovarian torsion: a case report and review of the literature. J Podiatry Surg. 2011 Dec; 46 (12): e19-23.

4. Jennifer Eng-Lunt, Heather Appelbaum, Jahn Avarello. Pediatric and adolescent ovarian torsion. ACEP news.

5. Kang HJ, Davis OK, Rosenwaks Z. Simultaneous bilateral ovarian torsion in the follicular phase after gonadotropin stimulation.www.fertstert.org/article/S0015 2006. 


\section{CASE REPORT}

\section{AUTHORS:}

1. Manohar R.

2. Gopalakrishna K. H.

3. Kavyashree G.

4. Kala B.

5. Karthik B.

\section{PARTICULARS OF CONTRIBUTORS:}

1. Assistant Professor, Department of Obstetrics and Gynaecology, Mandya Institute of Medical Sciences, Mandya.

2. Assistant Professor, Department of Surgery, Mandya Institute of Medical Sciences, Mandya.

3. Associate Professor, Department of Obstetrics and Gynaecology, Mandya Institute of Medical Sciences, Mandya.

4. Associate Professor, Department of Pathology, Mandya Institute of Medical Sciences, Mandya.
5. Senior Resident, Department of Surgery, Mandya Institute of Medical Sciences, Mandya.

\section{NAME ADDRESS EMAIL ID OF THE CORRESPONDING AUTHOR:}

Dr. Manohar R,

No. 2977, $8^{\text {th }}$ Cross,

Ashoknagar,

Mandya-571401.

Email: manoharrangaswamy@gmail.com

Date of Submission: 15/09/2014.

Date of Peer Review: 16/09/2014.

Date of Acceptance: 18/09/2014.

Date of Publishing: 25/09/2014. 